This item was submitted to Loughborough's Research Repository by the author.

Items in Figshare are protected by copyright, with all rights reserved, unless otherwise indicated.

\title{
Semiclassical quantization with bifurcating orbits
}

PLEASE CITE THE PUBLISHED VERSION

https://doi.org/10.1103/PhysRevA.66.033404

\section{PUBLISHER}

(c) The American Physical Society

\section{VERSION}

AM (Accepted Manuscript)

\section{PUBLISHER STATEMENT}

This work is made available according to the conditions of the Creative Commons Attribution-NonCommercialNoDerivatives 4.0 International (CC BY-NC-ND 4.0) licence. Full details of this licence are available at: https://creativecommons.org/licenses/by-nc-nd/4.0/

\section{LICENCE}

CC BY-NC-ND 4.0

\section{REPOSITORY RECORD}

Bartsch, Thomas, Jorg Main, and Gunter Wunner. 2019. "Semiclassical Quantization with Bifurcating Orbits". figshare. https://hdl.handle.net/2134/26785. 


\title{
Semiclassical quantization with bifurcating orbits
}

\author{
Thomas Bartsch, Jörg Main, and Günter Wunner \\ Institut für Theoretische Physik 1, Universität Stuttgart, 70550 Stuttgart, Germany
}

(Dated: February 3, 2008)

\begin{abstract}
Bifurcations of classical orbits introduce divergences into semiclassical spectra which have to be smoothed with the help of uniform approximations. We develop a technique to extract individual energy levels from semiclassical spectra involving uniform approximations. As a prototype example, the method is shown to yield excellent results for photo-absorption spectra for the hydrogen atom in an electric field in a spectral range where the abundance of bifurcations would render the standard closed-orbit formula without uniform approximations useless. Our method immediately applies to semiclassical trace formulae as well as closed-orbit theory and offers a general technique for the semiclassical quantization of arbitrary systems.
\end{abstract}

PACS numbers: 32.60.+i, 03.65.Sq, 31.15.Gy, 32.70.Cs

The correspondence between atomic spectra and classical orbits has been of fundamental interest and importance since the early days of quantum mechanics. The "old" quantum theory suffered from the severe drawbacks that the Bohr-Sommerfeld quantization rules can only be applied to integrable systems, and, for atomic systems, the Heisenberg principle for matrix elements is silent about transition amplitudes between low-lying and highly excited states. An important step towards a deeper understanding of the intimate connection between classical orbits and the quantum spectra was achieved by the development of periodic orbit theory [1] and, as a variant for the photo-excitation of atomic systems, closed orbit theory [2, 3]. In these theories, the density of states or photo-absorption spectra are given as the sum of two terms, one a smoothly varying part (as a function of energy) and the other a superposition of sinusoidal modulations. The frequencies, amplitudes, and phases of the modulations are directly given in terms of classical parameters of the orbits.

Closed-orbit theory has proven a powerful tool for the semiclassical interpretation of quantum spectra of, e.g., atoms in external magnetic and electric fields by explaining the peaks in the Fourier-transform recurrence spectra - qualitatively and even quantitatively - in terms of the

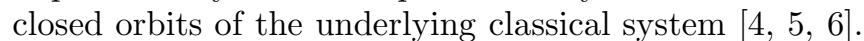
However, more than a decade after the development of closed-orbit theory the inverse procedure, i.e., the semiclassical calculation of the eigenenergies and transition amplitudes of individual states is still an unsolved problem. The reasons are twofold: Firstly, both closed-orbit and periodic-orbit theory suffer from fundamental convergence problems of the infinite orbit sums. Secondly, in generic systems the orbits undergo bifurcations when the energy is varied, and the semiclassical theories for isolated orbits exhibit unphysical divergences at the bifurcation points. Both problems have been addressed separately: Firstly, the harmonic inversion technique was introduced as a method for semiclassical quantization $[7,8$, which allows one to overcome the convergence problems of the closed orbit sum and to extract high-resolution spectra from a finite set of classical orbits. Secondly, in the vicinity of bifurcations the semiclassical approximation for isolated orbits was replaced with a uniform approximation describing all orbits involved in a bifurcation collectively 9, 10.

In this Letter both ideas are combined for the first time, i.e., we use both uniform approximations and harmonic inversion techniques for the semiclassical calculation of high-resolution spectra. In the presence of uniform approximations, the classical scaling laws which have been essential to all previous applications of the harmonic inversion technique [8] are no longer valid. Therefore, the harmonic inversion method must be generalized to handle the non-scaling functional form of uniform semiclassical approximations. It then gains a degree of flexibility in the quantization of arbitrary systems no other semiclassical quantization scheme has been able to reach to date.

The novel method will be demonstrated by way of example of the hydrogen atom in an electric field. As is well known, the classical dynamics of this system is integrable, which means that semiclassical energy eigenvalues can be calculated with the help of the EBKM torus quantization rules [11, 12]. However, when closed-orbit theory is applied the hydrogen atom in an electric field exhibits properties typical of mixed regular-chaotic systems as, e.g., Rydberg atoms in a magnetic field or Hénon-Heiles type systems. In particular, the closed orbits starting at and returning to the nucleus undergo bifurcations as the energy is varied. Contrary to the torus quantization, the method introduced in this Letter is not restricted to the Stark effect but can be applied to a large variety of systems with chaotic or mixed regular-chaotic classical dynamics. Furthermore, it can be used in connection with periodic-orbit theory [1] as well as closed-orbit theory.

The classical dynamics of the Stark system has already been discussed in detail 111. For any energy, the electron can go "uphill" against the direction of the electric field until the external field and the Coulomb field make it return to the nucleus. Alternatively, the electron can leave the nucleus in the "downhill" direction of the external field. The downhill orbit is closed only for energies below the Stark saddle point energy, $E_{\mathrm{S}}=-2 F^{1 / 2}$, otherwise 
the electron will cross the Stark saddle and escape to infinity. In addition to these axial closed orbits, there are non-axial orbits returning to the nucleus after $k$ oscillations in the downhill direction and $l>k$ oscillations in the uphill direction. Each of these orbits is generated in a bifurcation off the downhill orbit at a critical energy $E_{\text {gen }}$ and destroyed in a collision with the uphill orbit at $E_{\text {dest }}>E_{\text {gen }}$. Outside this energy range, they exist as complex "ghost" orbits.

Closed-orbit theory associates modulations observed in the quantum photo-absorption spectra of Rydberg atoms in external fields with the classical closed orbits. The quantum response function

$$
g(E)=\sum_{n} \frac{|\langle i|D| n\rangle|^{2}}{E-E_{n}+i \epsilon}=\left\langle i\left|D G_{E}^{+} D\right| i\right\rangle
$$

where $|i\rangle$ is the initial state, $D$ the dipole operator and $G_{E}^{+}$the retarded Green's function, is given as a smooth background plus an oscillatory closed-orbit sum [2, 3, 13]

$$
g^{\mathrm{osc}}(E)=\sum_{\mathrm{co}} \mathcal{A}_{\mathrm{co}}(E) e^{i S_{\mathrm{co}}(E)},
$$

where $S_{\text {co }}$ is the action of a closed orbit (co) and $\mathcal{A}_{\text {co }}$ a recurrence amplitude calculated from the monodromy matrix of the orbit and its initial and final directions with respect to the electric field. It includes a complex phase given by the Maslov index. In the following we are using atomic units, with $\hbar=1$ and $F_{0}=5.14 \times 10^{9} \mathrm{~V} / \mathrm{cm}$ the unit of the electric field strength.

The most convincing semiclassical interpretation of quantum spectra can be obtained by means of "scaled energy spectroscopy": By rescaling the classical quantities with suitable powers of, e.g., the electric field strength $F$, the classical dynamics can be shown not to depend on the energy $E$ and the field strength $F$ separately, but only on the scaled energy $\tilde{E}=E F^{-1 / 2}$. When recording quantum states at a fixed scaled energy $\tilde{E}$ as a function of the scaling parameter $w=F^{-1 / 4}$, each isolated closed orbit contributes a sinusoidal modulation to the sum (2), which can be extracted by a Fourier transform of the quantum spectrum. Experimental scaled energy spectra of atoms in electric fields have been analyzed in this way [5, 6]. The analysis reveals strong evidence for closed orbit bifurcations.

The simple semiclassical approximation embodied in the closed-orbit formula (2) fails close to a bifurcation of closed orbits, resulting in the divergence of the recurrence amplitudes. To overcome this difficulty, the closed-orbit terms for isolated orbits in (2) must be replaced with a uniform approximation describing all orbits involved in a bifurcation collectively. A uniform approximation suitable for regularizing the bifurcation of a non-axial orbit off either the downhill or the uphill orbit was derived by Gao and Delos 10] as well as Shaw and Robicheaux [14. We will use a slightly modified version of their result that gives the collective contribution of the axial and non-axial orbits participating in a bifurcation in terms of their actions $S_{\text {ax }}$ and $S_{\text {non }}$ and recurrence amplitudes $\mathcal{A}_{\text {ax }}$ and $\mathcal{A}_{\text {non }}$ as

$$
\Psi(E)=\left[\frac{\mathcal{A}_{\mathrm{non}}}{(1+i)} I+\frac{1}{a}\left(a \mathcal{A}_{\mathrm{ax}}+\frac{1+i}{\sqrt{2 \pi}} \mathcal{A}_{\mathrm{non}}\right)\right] e^{i S_{\mathrm{ax}}}
$$

where $I$ is given in terms of the standard Fresnel integrals $C(x)$ and $S(x)$ 15,

$$
I=e^{-i a^{2} / 4}\left[\frac{1+i}{2}-C\left(-\frac{a}{\sqrt{2 \pi}}\right)-i S\left(-\frac{a}{\sqrt{2 \pi}}\right)\right]
$$

and

$$
a= \pm 2 \sqrt{S_{\mathrm{ax}}-S_{\mathrm{non}}} .
$$

The negative sign for $a$ has to be chosen if the non-axial orbit is a complex ghost orbit.

The high-resolution quantization by harmonic inversion $[7,8]$ is based on the observation that by equating the quantum recurrence function (1) to its semiclassical approximation (2) - the smooth part can be neglected and taking the Fourier transform we obtain

$$
-i \sum_{n} d_{n} e^{-i E_{n} t}=C(t)
$$

with $d_{n}=|\langle i|D| n\rangle|^{2}$ and

$$
C(t)=\frac{1}{2 \pi} \int_{-\infty}^{\infty} d E \sum_{\text {со }} \mathcal{A}_{\mathrm{co}}(E) e^{i S_{\mathrm{co}}(E)} e^{-i E t} .
$$

The quantization problem has thus been recast as the problem of extracting the frequencies $E_{n}$ and amplitudes $d_{n}$ from a given time signal $C(t)$ of the form (6), provided the signal (7) can be calculated. In the case of a scaling system the signal is given as a sum of $\delta$ functions.

While the uniform approximation (3) successfully smoothes the divergences in (2), it spoils the classical scaling properties [14]. Therefore, the Fourier transform of a spectrum including uniform approximations cannot be evaluated in terms of $\delta$ functions. In fact, for nonscaling systems, there seems to be no way at all to compute the integral (7) because, apart from the fact that the classical quantities can always be calculated in a finite energy interval only, the integral can in general not even be expected to converge. Therefore, neither by analytical nor by numerical means will one be able to compute a useful semiclassical signal from (17). The inclusion of uniform approximations in semiclassical quantization is thus a nontrivial and challenging task.

To solve the problem we resort to the observation made in 16 that a band-limited signal, which only contains the spectral information describing the quantum system in a finite energy interval $\left[E_{\min }, E_{\max }\right]$ can be obtained by restricting the energy integral in (7) to this window. The resulting signal

$$
C^{\mathrm{bl}}(t)=\frac{1}{2 \pi} \int_{E_{\min }}^{E_{\max }} g^{\mathrm{osc}}(E) e^{-i E t} d E
$$



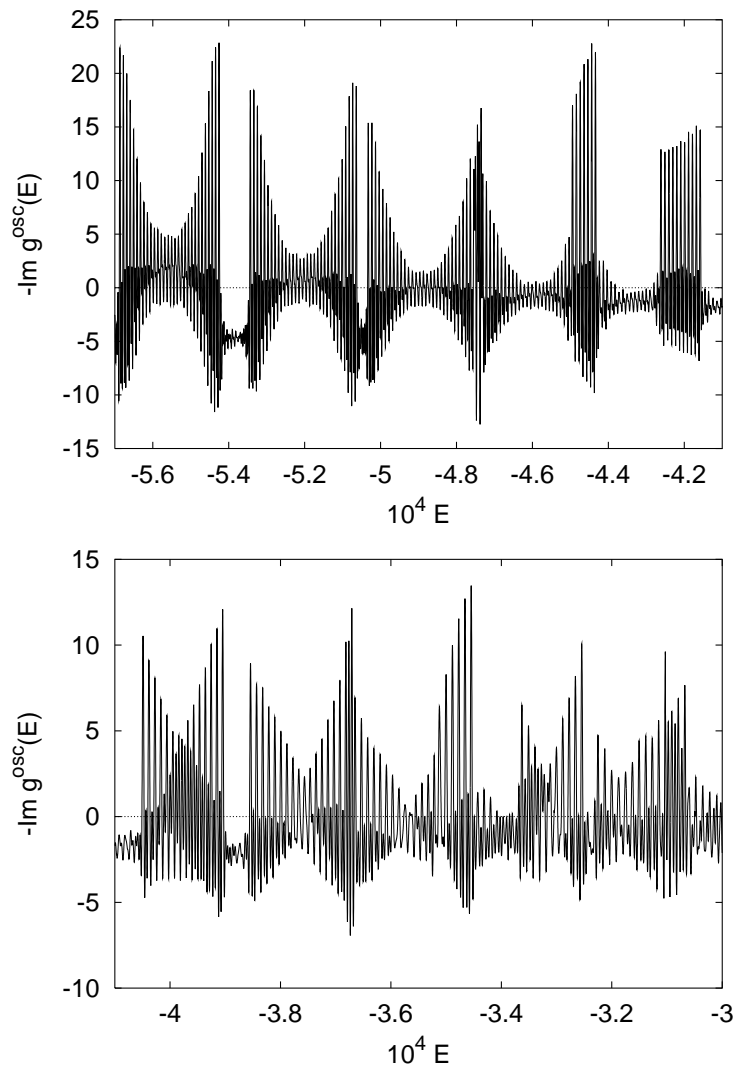

FIG. 1: Low-resolution semiclassical photo-absorption spectrum for the hydrogen atom in an electric field $F=51.4 \mathrm{~V} / \mathrm{cm}$ with initial state $|1 s 0\rangle$ and light polarized along the electric field axis. The scaled truncation time is $T_{\max }=15 \times 10^{6}$.

where $g^{\text {osc }}(E)$ is in general a non-scaling function that includes both contributions of isolated closed orbits and uniform approximations, can be evaluated numerically by standard methods. As it contains only a small number of frequencies in the interval chosen, it can be processed in a numerically stable way by conventional high-resolution methods such as linear prediction or Padé approximants. Contrary to previous methods for scaling systems, all of which contained the analytic evaluation of an integral, the numerical integration imposes no restrictions on the semiclassical response function occurring in the integrand. Notice that our method cannot be applied in connection with the original filter diagonalization algorithm [8]. It is only the separation between a low-resolution frequency filtering stage and a high-resolution harmonic inversion stage introduced in 16] that allows for the present generalization to arbitrary non-scaling semiclassical signals.

To demonstrate our method we investigate Stark spectra of the hydrogen atom for transitions from the ground state $|1 s 0\rangle$ to highly excited Rydberg states with light polarized parallel to the electric field axis. The external field strength is $F=10^{-8}$ a.u. $\hat{=} 51.4 \mathrm{~V} / \mathrm{cm}$. The high-resolution Stark spectrum is obtained in two steps:

Firstly, a low-resolution semiclassical spectrum is ob-
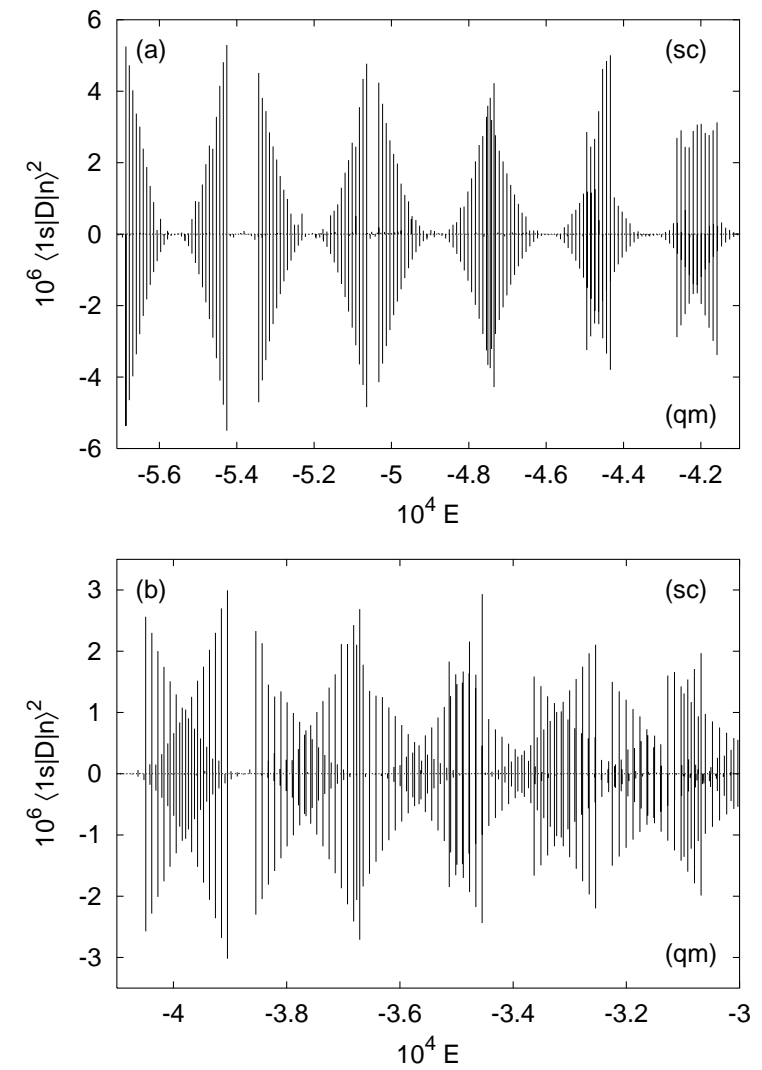

FIG. 2: High-resolution semiclassical (upper part) and quantum (lower part, inverted) photo-absorption spectrum for the hydrogen atom in an electric field $F=51.4 \mathrm{~V} / \mathrm{cm}$ with initial state $|1 s 0\rangle$ and light polarized along the electric field axis. The truncation time for the semiclassical spectrum is $T_{\max }=40 \times 10^{6}$.

tained by truncating the closed-orbit sum (2) at a maximal period $T_{\max }$. The cut-off value for the periods should not be chosen smaller than the signal length of the bandlimited signal (8) used for the harmonic inversion in the second step of the procedure. The low-resolution spectrum calculated with a scaled truncation time of $T_{\max }=15 \times 10^{6}$ is presented in Fig. 1. In the lower energy range shown in Fig. 1 (a), individual non-overlapping $n$-manifolds can be observed. (We have $n=30$ at $E \approx-5.56 \times 10^{-4}$.) In this region, the signal is sufficiently long to resolve individual spectral lines, although their precise determination from the plots remains difficult. In the higher energy range shown in Fig. 1)(b), two, three, or even four different $n$-manifolds overlap, leading to a drastically increased spectral density. In this region the semiclassical signal is evidently too short to discriminate individual lines. It is important to note that the possibility of computing the low-resolution spectrum depends critically on the use of uniform approximations. If it was calculated from isolated-orbit contributions only, a dense sequence of bifurcation-induced divergences would cover even the large-scale structure of the spectra. With the low-resolution semiclassical spectrum (Fig. 1) at hand 
the band-limited time signal ( 8 ) is now obtained by a numerical Fourier transform of $g^{\text {osc }}(E)$. In that calculation we used the signal length $T_{\max }=40 \times 10^{6}$ in order to resolve individual levels in the region of overlapping $n$ manifolds.

In the second step, the high-resolution semiclassical spectrum is finally obtained by harmonic inversion of the band-limited time signal (8). In Fig. 2 the semiclassical spectrum is compared to the exact quantum spectrum. The overall agreement between the semiclassical and the quantum spectrum is excellent, although for a few levels the comparison reveals discrepancies between the semiclassical and the quantum matrix elements. Note, in particular, the region of high spectral density at $E \approx 3.2 \times 10^{-4}$. In this region, groups of 3 nearly degenerate levels exist, some of which are well resolved semiclassically. At $E \approx-4.4 \times 10^{-4}$, even closer lines exist - they can hardly be discerned in the quantum spectrum. These lines are not resolved semiclassically. Instead, the harmonic inversion yields single lines with amplitudes equal to the sum of the two quantum amplitudes. We are confident to fully resolve even these states in the semiclassical spectrum when applying the cross-correlation technique for harmonic inversion [8].

In the future it should also be possible to extend the semiclassical spectrum to energies $E>E_{\mathrm{S}}$ where the classical motion is not completely bound, and to extract the semiclassical widths of the Stark resonances. A particular challenge is posed by the region around the Stark saddle point energy $E_{\mathrm{S}}$. Before the downhill orbit ceases to exist at $E_{\mathrm{S}}$, it undergoes an infinite sequence of bifur- cations, giving birth to non-axial orbits with arbitrarily high uphill repetition numbers. If subsequent bifurcations of a single orbit are too close, the uniform approximation (3) is no longer appropriate. It must then be replaced with a uniform approximation describing several bifurcations collectively. The uniformization of an infinite bifurcation cascade, in particular, remains an open problem whose solution is required to semiclassically cross the saddle point energy.

In summary, we have extended the harmonic inversion approach to semiclassical quantization to the quantization of systems without a scaling property. The generalized method allows for the inclusion of uniform approximations into the quantization procedure. We have demonstrated the effectiveness of our method by calculating a high-quality semiclassical spectrum for the hydrogen atom in an electric field in a spectral region where the semiclassical approximation without uniform approximations would be completely useless due to the abundance of bifurcations. With the modifications presented here, the technique of quantization by harmonic inversion has reached a stage where it does not impose any conditions on the classical dynamics of the system under study except that a semiclassical approximation to the response function can be given. Besides uniform approximations, any other non-standard semiclassical contributions such as diffractive corrections can be included. Thus, the harmonic inversion can now be regarded as a truly universal tool for the semiclassical quantization of arbitrary systems.
[1] M. C. Gutzwiller, Chaos in Classical and Quantum Mechanics (Springer, New York, 1990).

[2] M. L. Du and J. B. Delos, Phys. Rev. A 38, 1896 and 1913 (1988).

[3] E. B. Bogomolny, Sov. Phys. JETP 69, 275 (1989).

[4] J. Main, G. Wiebusch, K. Welge, J. Shaw, and J. B. Delos, Phys. Rev. A 49, 847 (1994).

[5] M. Courtney, H. Jiao, N. Spellmeyer, D. Kleppner, J. Gao, and J. B. Delos, Phys. Rev. Lett. 74, 1538 (1995).

[6] A. Kips, W. Vassen, and D. W. Hogervorst, Phys. Rev. A 59, 2948 (1999).

[7] J. Main, V. A. Mandelshtam, and H. S. Taylor, Phys. Rev. Lett. 79, 825 (1997).

[8] J. Main, Physics Reports 316, 233 (1999).

[9] J. Main and G. Wunner, Phys. Rev. A 55, 1743 (1997).
[10] J. Gao and J. B. Delos, Phys. Rev. A 56, 356 (1997).

[11] J. Gao and J. B. Delos, Phys. Rev. A 49, 869 (1994).

[12] V. Kondratovich and J. B. Delos, Phys. Rev. A 56, R5 (1997); 57, 4654 (1998).

[13] J. Gao, J. B. Delos, and M. Baruch, Phys. Rev. A 46, 1449 (1992).

[14] J. A. Shaw and F. Robicheaux, Phys. Rev. A 58, 1910 (1998).

[15] M. Abramowitz and I. A. Stegun, Pocketbook of Mathematical Functions (Verlag Harri Deutsch, Frankfurt/Main, 1984).

[16] J. Main, P. A. Dando, Dž. Belkić, and H. S. Taylor, J. Phys. A 33, 1247 (2000). 